Research Article

\title{
An Optimized Pulse-Counting Method for Compensation Vector Calculation in the Automatic Balancer
}

\author{
Lifang Chen $\mathbb{D}^{1},{ }^{1}$ Bo Zhou $\mathbb{D}^{2},{ }^{2}$ Zhaoju Li, ${ }^{1}$ Yixiang Guo, ${ }^{2}$ and Ziwen Yan $^{1}$ \\ ${ }^{1}$ Key Laboratory of Engine Health Monitoring-Control and Networking (Ministry of Education), \\ Beijing University of Chemical Technology, Beijing100029, China \\ ${ }^{2}$ Beijing Key Laboratory of Health Monitoring and Self-Recovery for High-End Mechanical Equipment, \\ Beijing University of Chemical Technology, Beijing 100029, China \\ Correspondence should be addressed to Lifang Chen; chenlf@mail.buct.edu.cn and Bo Zhou; 2018200652@mail.buct.edu.cn
}

Received 1 February 2020; Revised 3 May 2020; Accepted 11 May 2020; Published 31 May 2020

Academic Editor: Itzhak Green

Copyright (@ 2020 Lifang Chen et al. This is an open access article distributed under the Creative Commons Attribution License, which permits unrestricted use, distribution, and reproduction in any medium, provided the original work is properly cited.

\begin{abstract}
The accuracy of the counterweight positions in an automatic balancing system deeply affects dynamic balancing. Compensation vector is synthesized by the two counterweights located in the electromagnetic dual-weight automatic balancer. Therefore, if the position of the counterweight is inaccurate, it may result in a wrong adjustment and a larger imbalance of the rotor system. In this paper, an optimized pulse counting method for compensation vector calculation in an electromagnetic dual-weight balancing system is proposed based on a programmable logic controller (PLC). A propeller automatic balancing simulation test bench is used to verify the effect of the method by obtaining the positions of the counterweights and synthesizing the compensation vector in the working mode. The error is less than $1 / 80$ which means that it does not exceed one step in the 80 -position-balancer at 1200 rpm. The proposed control system can work without computers or high-speed data acquisition equipment, which improves the stability and flexibility of the control system, facilitates the design of the automatic balancing system, and shows excellent potential for industrial applications.
\end{abstract}

\section{Introduction}

More than $70 \%$ of the vibration failures of rotating machinery are caused by the imbalance-induced vibration of the rotor system [1]. Many balancing procedures have been developed to suppress this imbalance-induced vibration [2-10]. Dynamic balancing of the rotor system is usually achieved by starting and stopping the device and applying test weights and counterweights. An automatic balancing system changes the position of the counterweights on a given plane in real time to form the compensation vector. This approach has the significant advantage of being able to apply the counterweights without stopping the equipment and has been increasingly used in the industrial field [11-22], such as for high-precision machine tools for civilian use, grinders, and military turboprop automatic balancing systems.

In an electromagnetic automatic balancing system $[23,24]$, the compensation vector is generated by two independently rotating counterweights pre-installed on the rotor. Compared with a laser adding mass or removing mass balancing device, the structure is simpler, and no pollution and fatigue damage will occur, but the two counterweights on the circumference of the counterweight disk need to be accurately positioned during balancing to obtain an accurate compensation vector. The accuracy of the compensation vector will seriously affect the vibration suppression result. If the position error is larger than one position, it will result in wrong adjustment and a larger imbalance of the rotor system. In this paper, we propose a method for the accurate measurement of the counterweight position in an automatic balancing system based on a programmable logic controller (PLC). The system does not require a computer or highspeed data acquisition equipment, which greatly improves the stability and flexibility of the control system. The proposed method allows for the precise calculation of the compensation vector during automatic balancing. 


\section{Measurement Principle of Compensation Vector}

2.1. Structure of the Automatic Balancing System. The electromagnetic automatic balancing head includes two parts: a moving ring and a static ring. The three-dimensional structural diagram is shown in Figure 1. The moving ring is installed on the rotating shaft and rotates with the shaft, and the static ring is attached to the base with a bracket. Two counterweights with a certain mass are installed at a certain radius on the counterweight disk of the moving ring. The size of the eccentric mass on each counterweight disk depends on structure, force analysis of counterweight disks, and mainly the balancing capacity requirements of rotors. Once the installation radius of counterweights is determined, the mass of the counterweight on each disk can be calculated. The maximum balancing capacity of the automatic balancer used in this article is $18 \mathrm{~g} \times \mathrm{m}$. During the rotation, each counterweight will generate a compensation vector. The different angles of the two counterweights result in compensation vectors of different sizes and directions. A ring of magnets of opposite polarity is installed on the outer edge of the counterweight disk, and the counterweight is driven by the electromagnetic force generated by the static ring. When the counterweight is at the specified position, its position is locked by the self-locking force created by the magnet.

A positioning magnet for real-time monitoring of the position of the counterweight is installed on the end cover of the moving ring and the outer edges of the two counterweight disks. At the corresponding position of the static ring, three Hall sensors are used to detect the signals of the three positioning magnets. The three Hall sensors are arranged in a straight line in the axial direction and parallel to the rotor axis. The main body of the static ring has two electromagnetic coils. When the coil is energized, an electromagnetic field is formed between the moving ring and the static ring. The electromagnetic field changes regularly and interacts with the magnetic field of the magnet on the counterweight disk, causing the counterweight to rotate relative to the shaft. The target position of the counterweight is specified by the direction of rotation and the number of steps.

The whole auto-balancer system includes two modules, the vibration measuring module and the balancing actuator module. The first module can provide the compensation mass based on the influence coefficient method (ICM) with the vibration vector, and then convert to the two counterweight positions. The other module, balancing actuator, is designed with two mobile weights providing 0 to $2 \mathrm{MR}$ compensation ability based on the requirement of the rotor system. By detecting the vibration vector in real time, the required compensation weight is calculated by ICM and then converted to the position of two counterweights. Then optimal movement path of counterweights is selected so that the combined compensation vector generated by the two counterweights is opposite to the original mass imbalance vector of the rotor system. This is achieved using an errorfree algorithm to ensure real-time suppression of the vibration $[3,4,16,20,25,26]$. The vibration suppression effect of the balance head during normal movement of the counterweight is shown in Figure 2(a). If the position of the counterweight is incorrect during its movement, the counterweight will move in the opposite direction and increase the vibration, as shown in Figure 2(b).

Due to the principle of electromagnetic-driven step by step with alternating positive and negative pulses, the location of two counterweights will determine the direction of the next step and influence the balancing effect. Thus, accurate counterweights positions are crucial for the balancing actuator. The counterweights must be accurately positioned to ensure accurate movement and generate the required compensation vector. At present, most automatic balancing systems used for high-speed rotating machinery adopt highspeed data acquisition equipment to acquire the position signal of the internal counterweight. However, high-speed data acquisition equipment requires a computer for signal acquisition and calculations. However, other devices such as aircraft engines often carry a limited amount of weight, which needs a lightweight balancer. Therefore, it is necessary to design a simple and lightweight balancer control system that does not rely on a big size of data acquisition device and host computer.

\subsection{Principles of the Calculation of the Compensation Vector.} High-speed data acquisition equipment (such as NI highspeed acquisition equipment) and host computer acquisition software (such as LabVIEW software) are usually used to detect and analyze high-speed pulse signals which have big size and thereby limit industrial applications. Therefore, in this study, we develop a low-cost, highly stable, and simpleto-operate counterweight position detection system based on a PLC. A PLC is a control system specifically designed for application in industrial environments. It has the advantages of high reliability, easy programming, convenient installation, and flexible configuration. A PLC can be used without a computer by using a touch screen and other equipment.

The counterweight position detection and control system is shown in Figure 3. A reference Hall sensor and two position Hall sensors collect the signals of three magnets respectively, calculate the relative positions of the two counterweights on the balancer, and provide feedback information to form a closed-loop control system of the PLC controller and balancing actuator. Due to the different angles between the magnets on the two disks and the reference magnet, the Hall signals of the two disks and the reference Hall signals have different time intervals. The controller starts timing as soon as it detects the reference Hall signal and saves the current value of timer when it detects the Hall signal of each disk. By comparing these two times with the time taken for each revolution, that is, the time interval between two reference Hall signals, the current position of these two disks can be known. The arrangement of the counterweight position sensors and the time sequence of the signals are shown in Figure 4, and the position calculations are shown in Figure 5. The positions of the two 


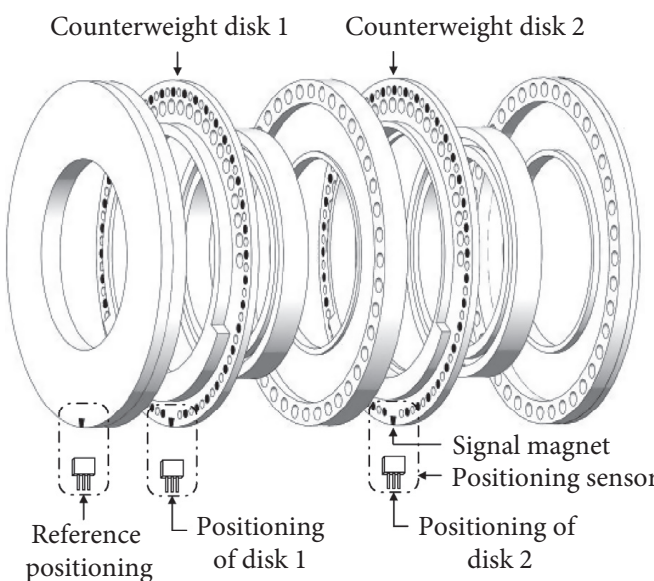

(a)

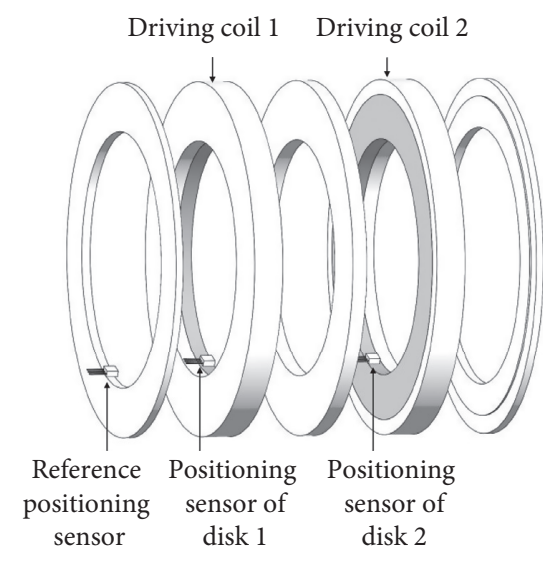

(b)

FIgURE 1: Schematic diagram of the balance head of the system. (a) Moving ring. (b) Static ring.

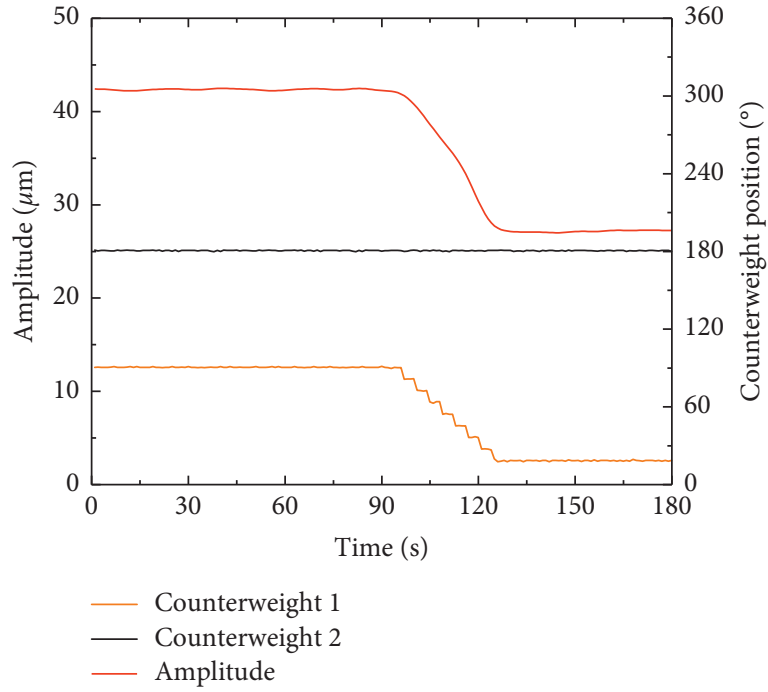

(a)

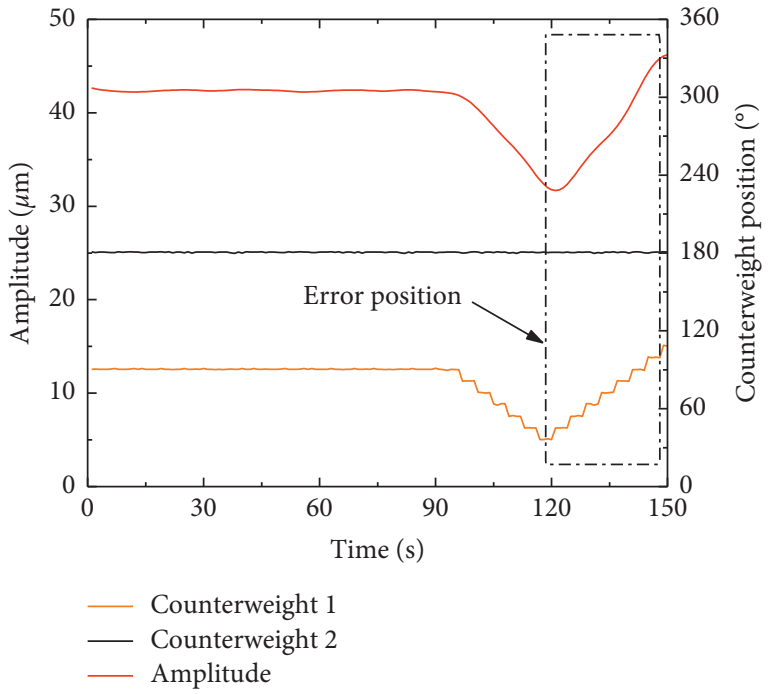

(b)

FIGURE 2: Schematic diagram of the vibration suppression effect of the balance head.

counterweights can be calculated based on the positions of the two disc signals in one rotation cycle.

For a given diameter of one actuator, the more positions the counterweights have, the higher the balancing accuracy, and the more difficult it is to obtain stable counterweights positions in the case of high-speed rotor balancing. For the balancer used in this article, the maximum number of magnets that can be accommodated in this structure is 80 . So the counterweight of the balancer used in the experiment has a total of 80 steps to ensure maximum accuracy; therefore, the step angle is $4.5^{\circ}$. The stability of the counterweight position is very important for the movement of the counterweight of the balance head. Even an error of one step will cause incorrect movement of the counterweight, preventing accurate balancing, and the vibration of the shaft may increase, which is dangerous. Therefore, the results of the position detection of the two counterweights must be stable, and the error of the counterweight position cannot be more than one step.

\section{Optimized Pulse Counting Method for Calculating the Compensation Vector}

3.1. Timing Method. When the reference magnet passes the Hall sensor, a pulse signal is generated, and the timer inside the PLC begins counting. When the reference magnet passes the Hall sensor again, the current value is $T$, i.e., the time it takes the rotor to make one revolution is $T$ (unit: seconds). The speed of the rotor (unit: rpm) is defined as

$$
v=\frac{60}{T} \text {. }
$$




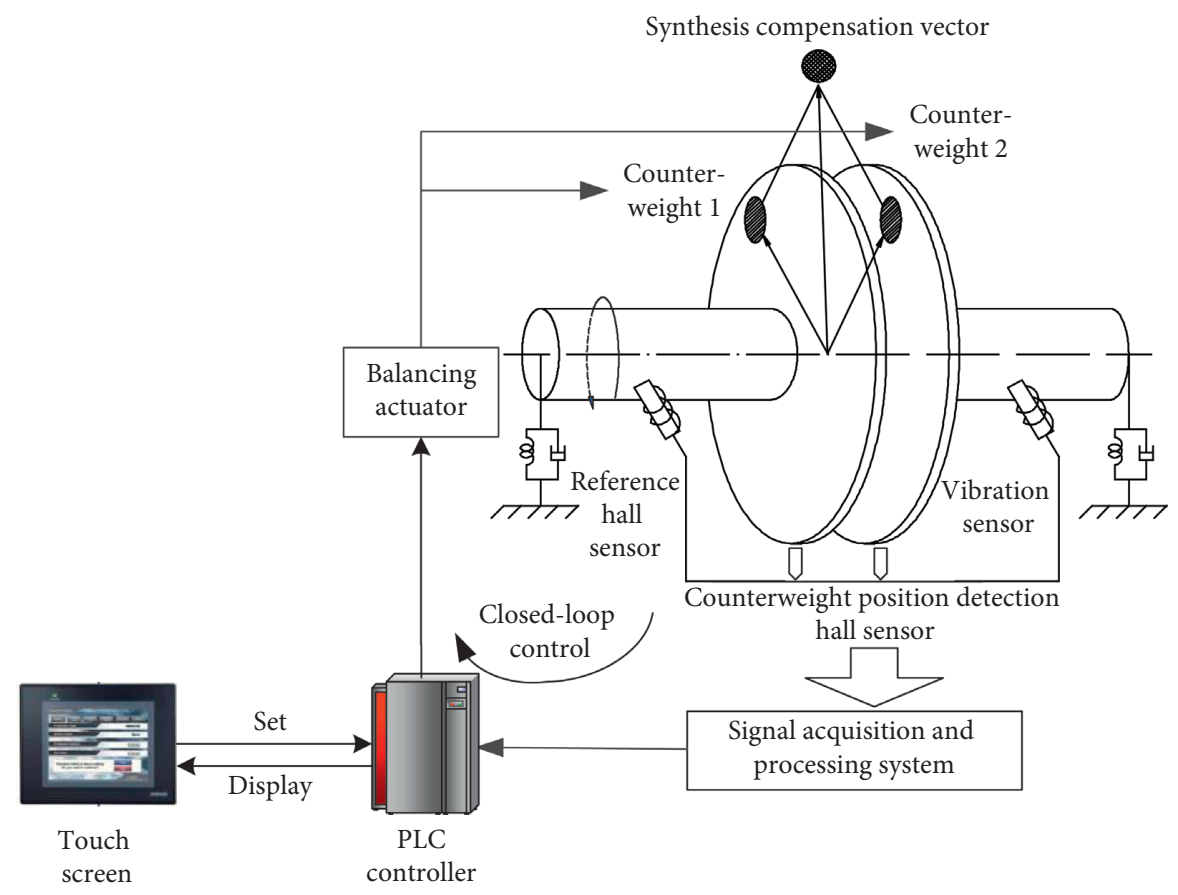

FIgURe 3: Position detection and control system of the counterweight.

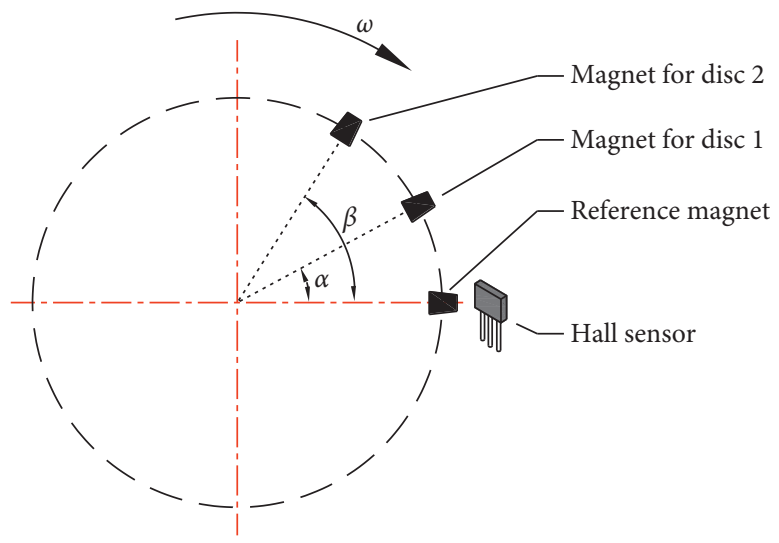

(a)

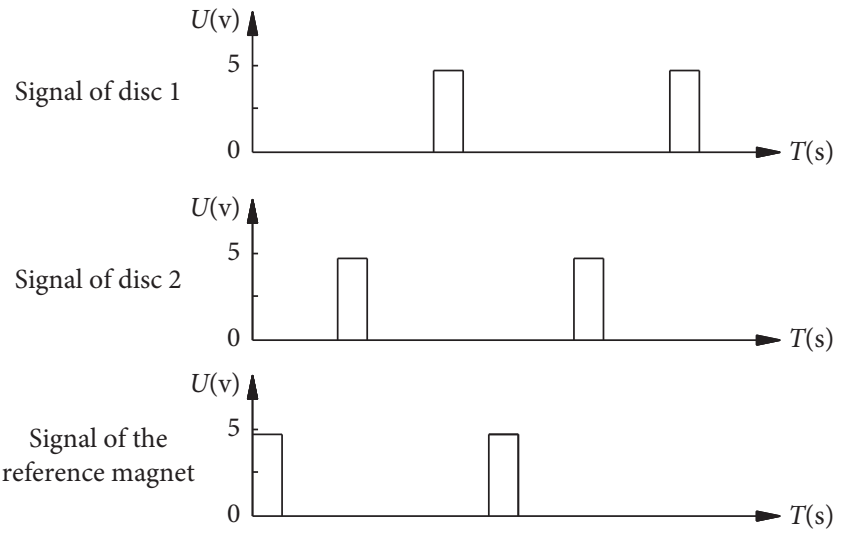

(b)

FIGURe 4: Principle of the position detection of the counterweight. Here, $\alpha$ and $\beta$ represent the angle between the positioning magnet and the reference magnet of disc 1 and disc 2 , respectively, in radians; $\omega$ represents the angular velocity of the rotating shaft, in radians per second; $U$ represents the voltage output by sensors in volts; and T represents time in seconds.

During one rotation of the rotor, the other two Hall sensors receive the signals of the corresponding magnets and record the current values ( $T_{1}$ and $T_{2}$, in seconds) at the same time. The current positions of the two counterweights are defined as

$$
\begin{aligned}
& L_{1}=\frac{T_{1}}{T} \times 80, \\
& L_{2}=\frac{T_{2}}{T} \times 80 .
\end{aligned}
$$

The key to the accurate calculation of the position is the precise measurement of $T, T_{1}$, and $T_{2}$. This measurement method does not require high-precision measurement of time; therefore, the high-speed pulse function of the PLC can be used because it has sufficient accuracy.

3.2. Pulse-Counting Method. The PLC generates highspeed pulses at the specified frequency in a stable manner. The pulse frequency is $f$, the number of pulses corresponding to one rotation of the rotor is $n$, and the number of pulses between the reference signal and the Hall signals of the two counterweights is $n_{1}$ and $n_{2}$. The speed is defined as

$$
v=\frac{60 \times f}{n}
$$




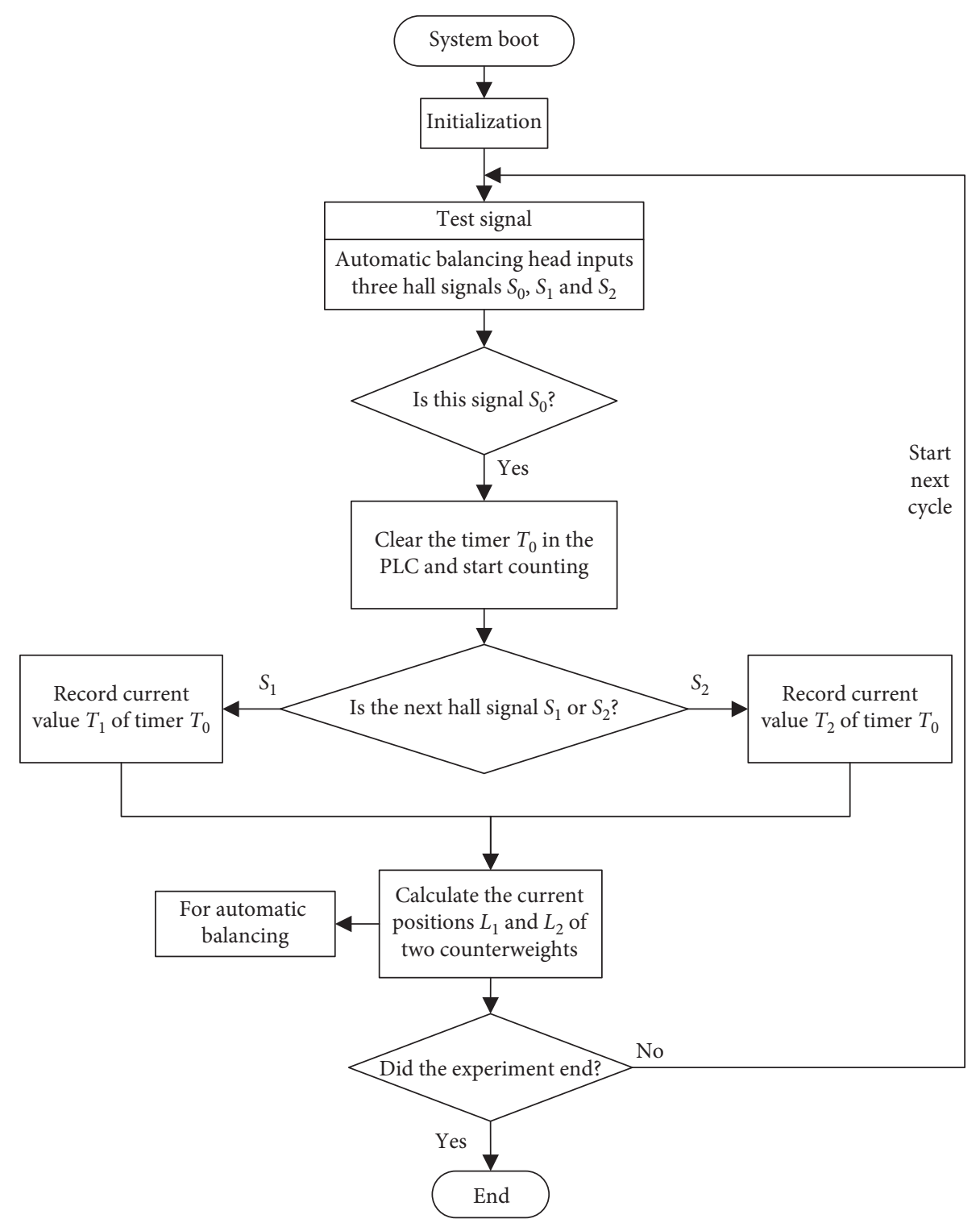

FIGURE 5: Flowchart for calculating the counterweight position. Here, $\mathrm{S}_{0}$ represents the signal of the reference Hall, $\mathrm{S}_{1}$ represents the Hall signal of the disk 1 , and $\mathrm{S}_{2}$ represents the Hall signal of the disk 2.

The current positions of the two counterweights are defined as

$$
\begin{aligned}
& L_{1}^{\prime}=\frac{n_{1}}{n} \times 80, \\
& L_{2}^{\prime}=\frac{n_{2}}{n} \times 80 .
\end{aligned}
$$

When an external disturbance causes $n_{1}$ and $n_{2}$ to fluctuate with a value of $\Delta n$, the error associated with the calculation of the two counterweights is expressed as

$$
\begin{aligned}
& \frac{L_{1}^{\prime \prime}-L_{1}^{\prime}}{L_{1}^{\prime}}=\frac{\left(\left(n_{1}+\Delta n\right) / n\right) \times 80-\left(n_{1} / n\right) \times 80}{\left(n_{1} / n\right) \times 80}=\frac{\Delta n}{n_{1}}, \\
& \frac{L_{2}^{\prime \prime}-L_{2}^{\prime}}{L_{2}^{\prime}}=\frac{\left(\left(n_{2}+\Delta n\right) / n\right) \times 80-\left(n_{2} / n\right) \times 80}{\left(n_{2} / n\right) \times 80}=\frac{\Delta n}{n_{2}} .
\end{aligned}
$$

Therefore, the size of $n_{1}$ and $n_{2}$ is related to the pulse frequency $f$. It is evident that an increase in the pulse frequency reduces the random error, but at a high pulse frequency, noise interference will occur and the stability is reduced; therefore, the pulse frequency should not be too high.

3.3. Optimized Pulse-Counting Method. During the rotation of the rotor, the sensor signal will generate random fluctuations due to interference by environmental factors. The least-squares regression equation is defined as

$$
\begin{aligned}
& a=\frac{\sum x y-(1 / N) \sum x \sum y}{\sum x^{2}-(1 / N)\left(\sum x\right)^{2}}=\frac{\sum i L_{i}-(1 / n) \sum i \sum L_{i}}{\sum i^{2}-(1 / n)\left(\sum i\right)^{2}}, \\
& b=\bar{y}-a \bar{x}=\overline{L_{i}}-a \bar{i} .
\end{aligned}
$$


In equation (6), the serial number $i$ is taken as the abscissa $x$ and the calculated position of the disk is taken as the ordinate $y$. After curve fitting, the regression line must pass through the average values $\left(\bar{i}, t \overline{L_{i}}\right)$. If the average number of data is $n$, and there are $x(x<n / 2)$ calculation results with a total error of $\Delta n$, the relative error after averaging is defined as

$$
\frac{\Delta n}{n}<\frac{\Delta n}{x} \times \frac{1}{2}
$$

The error of each position calculation result after averaging is less than half of the average error of each position result before averaging. When the average error of each position result before averaging is $4.5^{\circ}$, each position result after averaging produces an error of less than $2.25^{\circ}$. Therefore, the averaging process reduces the error to an acceptable value and eliminates the error caused by environmental interference.

\section{Experimental Research}

4.1. Simulation Test. The step angle of the automatic balancing head used in the experiment is $4.5^{\circ}$. In the simulation test, we set the Hall signal of disc 1 to lag the reference Hall signal by $45^{\circ}$. LabView software is used to simulate the reference Hall signal and the dual-disc Hall signal and calculate the positions, when the Hall sensor waveform generates a pulse falling edge and the voltage is below -4 volts, the program will treat it as a signal. The analog signal obtained from the simulation is shown in Figure 6. Each prominent spike in the figure represents a Hall signal. The two analog signals have the same white noise and one superposition of square waves with different phases, which is similar to the actual Hall signal. For example, in disk 1, when the calculated value of the angle of disk 1 fluctuates by more than half of the step angle, i.e., when the error exceeds $\pm 2.25^{\circ}$, an error of one step occurs between the calculated result of the position of disk 1 and the actual position. In the simulation experiment, the phase difference between the disc 1 signal and the reference signal is $45^{\circ}$, corresponding to the 10th step position of disc 1 . The simulation results are shown in Figure 7(a). It is observed that the pulse-counting method results in three sudden changes in the calculated position from $45^{\circ}$ to $40.5^{\circ}$ in 200 runs of the simulation after rounding the degrees to a multiple of 4.5 (the step interval of balancer is $4.5^{\circ}$ ). The calculated position of disc 1 deviates from the actual position. The calculation results are not sufficiently stable, and this scheme requires improvement.

During the rotation of an actual rotor, the signal of the sensor is disturbed by many factors, for example, a complex electromagnetic environment, extra vibration caused by the movement of the human body, and the passing of nearby cars and subways, etc., generating random fluctuations, which cause position errors. The noise signal added in the simulation test is used to simulate these disturbances. The values of the position of the disk 1 are averaged to

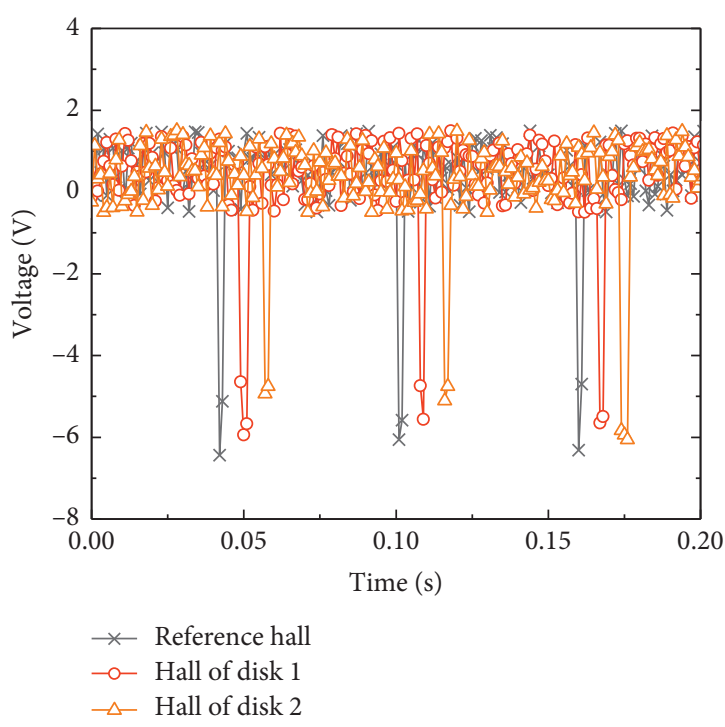

Figure 6: Simulation signal.

eliminate the errors caused by the fluctuations, i.e., the average of 50 position results adjacent to the current position on disc 1 is obtained. The result is shown in Figure $7(\mathrm{~b})$. The average angle does not fluctuate more than $\pm 2.25^{\circ}$ (the data of Figure 7 (b) are not rounded to a multiple of 4.5. The purpose is to show that the data fluctuation is very small. If rounded, there is only one straight line on this graph at $45^{\circ}$ ). The positioning result is stable at $45^{\circ}$, and the counterweight position is stable and accurate in this scheme.

4.2. Propeller Test on an Automatic Balance Test Bench. The PLC (Omron CP1H) is the core equipment of the control system. The automatic balance test bench for the test is shown in Figure 8. The PLC-based process for calculating the counterweight position is shown in Figure 5. The pulse frequency is set to $20 \mathrm{kHz}$ according to the accuracy requirements.

In the experiment, the counterweight 1 is fixed at the position of Step 24. Table 1 shows the number of pulses in a given period, and Figure 9 shows the position result of the pulse-counting method. The total number of pulses in the fourth circle is 990 . The position of the counterweight 1 corresponds to 292 pulses and is defined as

$$
L_{1}^{\prime}=\frac{T_{1}}{T} \times 80=\frac{292}{990} \times 80=23.596(\text { step }) .
$$

Because the number of steps must be an integer, the result is rounded to 24 . However, the total number of pulses in the fifth circle is 993 and the number of pulses corresponding to the counterweight 1 is 290 . In this case, the position of counterweight 1 is defined as

$$
L_{1}^{\prime}=\frac{T_{1}}{T} \times 80=\frac{290}{993} \times 80=23.364(\text { step }) .
$$




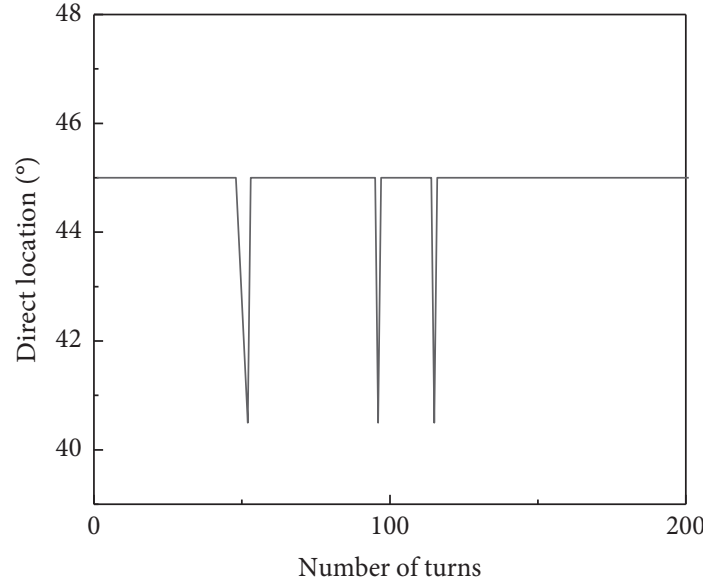

(a)

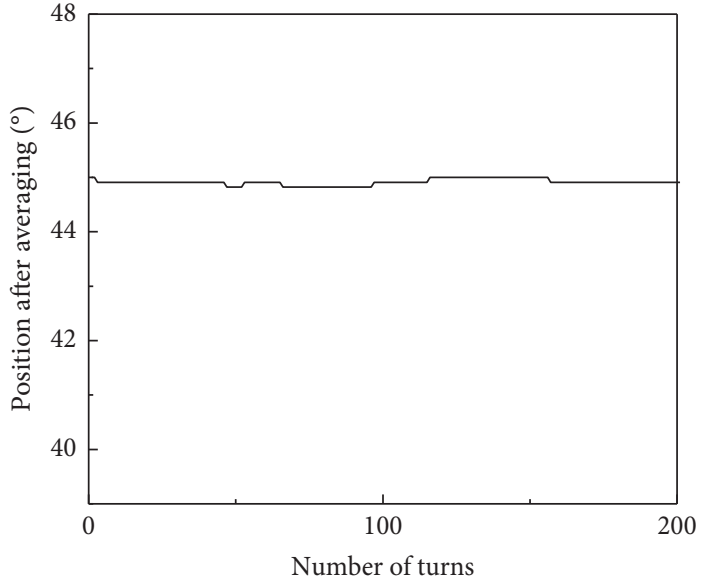

(b)

FIGURE 7: Simulation test results.

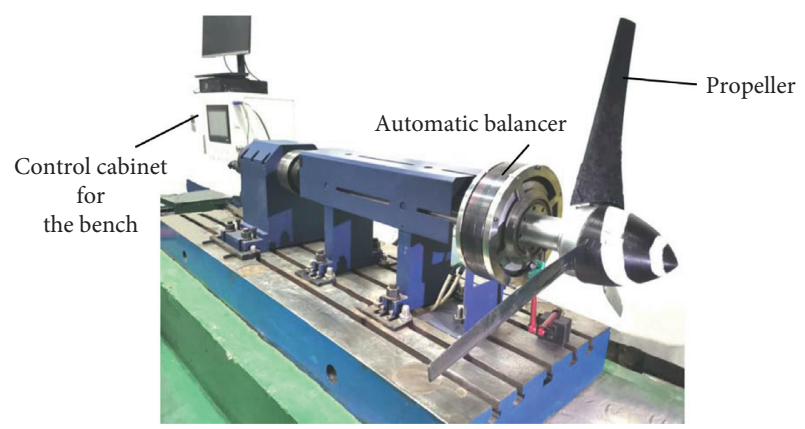

Figure 8: Propeller simulation automatic balance test bench.

TABLE 1: Experimental data of the pulse-counting method.

\begin{tabular}{lcccc}
\hline $\begin{array}{l}\text { Seri. } \\
\text { Unit }\end{array}$ & $\begin{array}{c}\text { One cycle } \\
\text { Pulse }\end{array}$ & $\begin{array}{c}\text { Disk 1 } \\
\text { Pulse }\end{array}$ & $\begin{array}{c}\text { Position } \\
\text { Step }\end{array}$ & $\begin{array}{c}\text { Rounded position } \\
\text { Step }\end{array}$ \\
\hline 1 & 991 & 292 & 23.572 & 24 \\
2 & 991 & 292 & 23.572 & 24 \\
3 & 990 & 292 & 23.596 & 24 \\
4 & 990 & 292 & 23.596 & 24 \\
5 & 993 & 290 & 23.364 & 23 \\
6 & 988 & 293 & 23.725 & 24 \\
7 & 988 & 293 & 23.725 & 24 \\
\hline
\end{tabular}

After rounding, the current position is Step 23, which is one step less than the position result of the previous circle. This error does not meet the accuracy requirement.

The average of the multiturn pulse data is used to eliminate fluctuations in the calculated position. After analyzing the experimental data of the pulse counting scheme, it was found that the fluctuation of the position was primarily due to the external disturbances. When fluctuations occur in the pulses intercepted by the reference Hall sensor and the counterweight Hall sensor at the same time, the position of the counterweight will fluctuate. After repeated

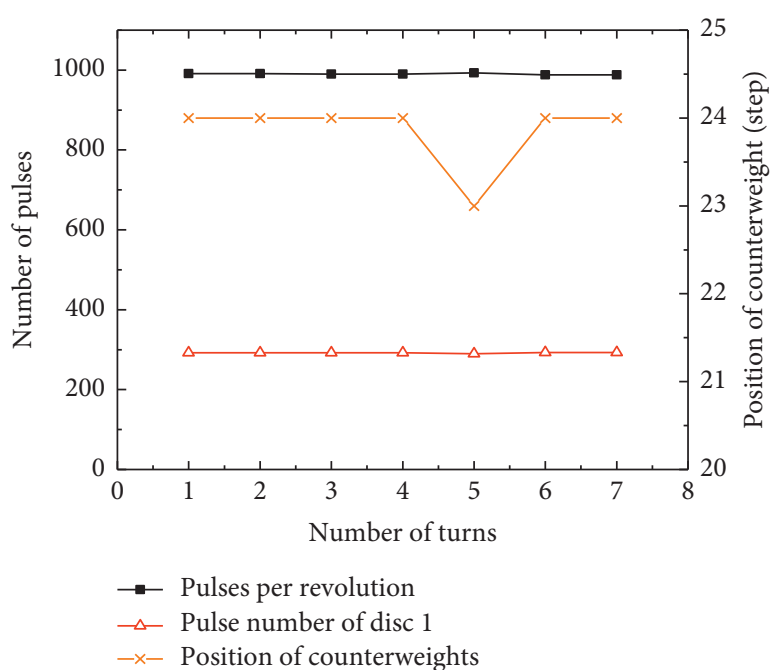

Figure 9: Position result of the pulse-counting method.

TABle 2: Experimental data of the optimized pulse-counting method.

\begin{tabular}{lcccc}
\hline $\begin{array}{l}\text { Seri. } \\
\text { Unit }\end{array}$ & $\begin{array}{c}\text { One cycle } \\
\text { Pulse }\end{array}$ & $\begin{array}{c}\text { Disk 1 } \\
\text { Pulse }\end{array}$ & $\begin{array}{c}\text { Position } \\
\text { Step }\end{array}$ & $\begin{array}{c}\text { Rounded position } \\
\text { Step }\end{array}$ \\
\hline 1 & 988.8 & 292.1 & 23.632 & 24 \\
2 & 988.8 & 292.1 & 23.632 & 24 \\
3 & 988.8 & 292.1 & 23.632 & 24 \\
4 & 988.8 & 292.1 & 23.632 & 24 \\
5 & 989.0 & 291.4 & 23.571 & 24 \\
\hline
\end{tabular}

experiments, it is found that averaging every 50 points will stabilize the position detection result of the counterweight and provides the required positional accuracy. Table 2 shows part of the data of the position of the counterweight $1 \mathrm{after}$ averaging, and Figure 10 shows the position result of the optimized pulse-counting method. The calculation result of the counterweight position is stable. 


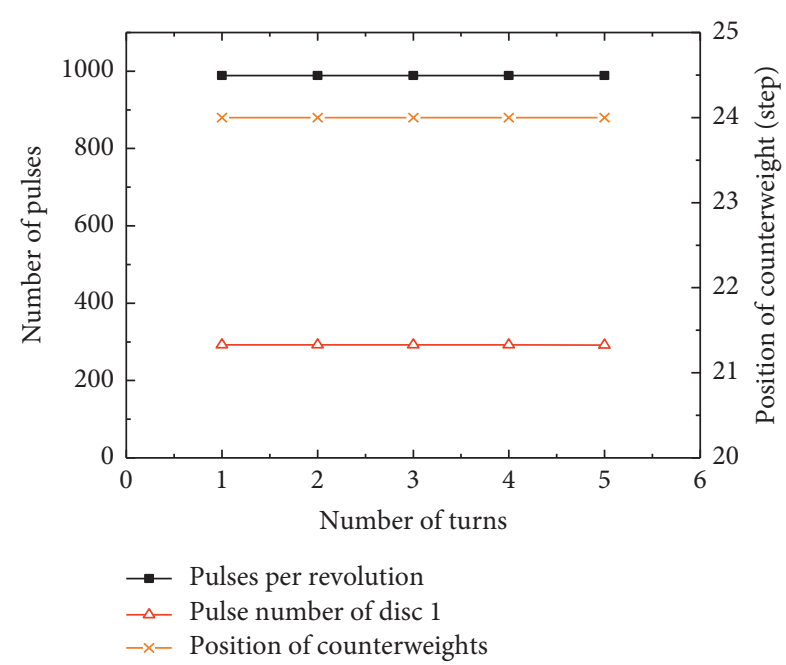

FIGURE 10: Position result of the optimized pulse-counting method.

\section{Summary and Outlook}

We developed an optimized pulse counting for the accurate measurement of the position of the counterweights in automatic balancing based on PLC. The results showed that the proposed method is feasible, and the position results are stable in the error analysis and algorithm correction. The proposed method provides the same performance as highspeed data acquisition equipment with an acceptable error. The following conclusions are drawn:

(1) This study only verified the principle of the method under laboratory conditions. In future studies, it is necessary to optimize the hardware configuration and method in an actual working environment with interference (such as an environment with alternating electromagnetic fields and variable speed).

(2) Because of the good performance of the PLCbased method for electromagnetic automatic balancing, it is necessary to consider incorporating vibration monitoring into the position detection program in subsequent studies to improve the performance of the automatic balancing control system.

(3) For flexible rotors, the balancer can also be used to add the compensation vector, but different rotors have different modals at different speeds, so the structure and installation position of the balancer should be rationally optimized to adapt to this working condition.

(4) The PLC-based counterweight position detection system does not require a computer and high-speed data acquisition equipment, which simplifies the control system structure and allows for the integration of position monitoring and drive control. The stability of the system also reduces the cost of the control system, which is valuable in industrial applications.

\section{Data Availability}

The raw/processed data required to reproduce these findings cannot be shared at this time as the data also form part of an ongoing study.

\section{Conflicts of Interest}

The authors declare that they have no conflicts of interest.

\section{References}

[1] Y. C. Luo, Research of Active Balancing Control System of Rotor Based on Vibration Signal Extraction, Hefei University of Technology, HeFei, China, 2015.

[2] R. H. Pessrkinson and D. R. Danielson, Simplified Propeller Balancing System and Method, US Patents, Alexandria, VA, USA, 2016.

[3] C.-J. Lu and M.-H. Tien, "Pure-rotary periodic motions of a planar two-ball auto-balancer system," Mechanical Systems and Signal Processing, vol. 32, pp. 251-268, 2012.

[4] J. Ehyaei and M. M. Moghaddam, "Dynamic response and stability analysis of an unbalanced flexible rotating shaft equipped with n automatic ball-balancers," Journal of Sound and Vibration, vol. 321, no. 3-5, pp. 554-571, 2009.

[5] S. N. Mahmoodi, M. J. Craft, S. C. Southward, and M. Ahmadian, "Active vibration control using optimized modified acceleration feedback with Adaptive Line Enhancer for frequency tracking," Journal of Sound and Vibration, vol. 330, no. 7, pp. 1300-1311, 2011.

[6] F. Beltrán-Carbaja and G. Silva-Navarro, "Active vibration control in duffing mechanical systems using dynamic vibration absorbers," Journal of Sound and Vibration, vol. 333, no. 14, pp. 3019-3030, 2014.

[7] X. Yu, K. Mao, S. Lei, and Y. Zhu, "A new adaptive proportional-integral control strategy for rotor active balancing systems during acceleration," Mechanism and Machine Theory, vol. 136, pp. 105-121, 2019.

[8] T. Messager and M. Pyrz, "Discrete optimization of rigid rotor balancing," Journal of Mechanical Science and Technology, vol. 27, no. 8, pp. 2231-2236, 2013.

[9] Z. Shihai and Z. Zimiao, "Research on the field dynamic balance technologies for large diesel engine crankshaft system," Shock and Vibration, vol. 2017, Article ID 7150472, 10 pages, 2017.

[10] W. C. Foiles, P. E. Allaire, and E. J. Gunter, "Review: rotor balancing," Shock and Vibration, vol. 5, no. 5-6, pp. 325-336, 1998.

[11] X. Pan, H. Q. Wu, J. J. Gao, and W. M. Wang, "New liquid transfer active balancing system using compressed air for grinding machine," Journal of Vibration and Acoustics, vol. 137, no. 1, 2015.

[12] B. Hredzak and G. Guo, "New electromechanical balancing device for active imbalance compensation," Journal of Sound and Vibration, vol. 294, no. 4-5, pp. 737-751, 2006.

[13] J. Su and B. Gao, "A new type of auto-balance device for grinding wheel," Applied Mechanics and Materials, vol. 66-68, pp. 1743-1747, 2011.

[14] W. L. Winzenz, D. Boswell, A. Badre-Alam, and D. Morris, Aircraft Propeller Balancing System, US Patents, Alexandria, VA, USA, 2015.

[15] R. Brian, R. M. Paul, and S. B. J. C Church, "C-130J-30 inflight propeller balancing," in Proceedings of the 7th DSTO 
International Conference on Health \& Usage Monitoring (HUMS 2011), Melbourne, Australia, March 2011.

[16] Y. M. Huang and G. H. Tarng, Method for Controlling Ball Auto-Balance System of Optical Disk Drive, US Patents, Alexandria, VA, USA, 2010.

[17] B. G. Bykov, "Auto-balancing of a rotor with an orthotropic elastic shaft," Journal of Applied Mathematics and Mechanics, vol. 77, no. 4, pp. 369-379, 2013.

[18] T. Kim and S. Na, "New automatic ball balancer design to reduce transient-response in rotor system," Mechanical Systems and Signal Processing, vol. 37, no. 1-2, pp. 265-275, 2013.

[19] A. Blanco-Ortega, G. Silva-Navarro, J. Coln-Ocampo, M. Oliver-Salazar, and G. Vela-Valds, "Automatic balancing of rotor-bearing systems," in Advances on Analysis and Control of Vibrations-Theory and Applications, Intechopen Limited, London, UK, 2012.

[20] D. J. Rodrigues, A. R. Champneys, M. I. Friswell, and R. E. Wilson, "Automatic two-plane balancing for rigid rotors," International Journal of Non-linear Mechanics, vol. 43, no. 6, pp. 527-541, 2008.

[21] B. Dyniewicz, A. PreGowska, and C. I. Bajer, "Adaptive control of a rotating system," Mechanical Systems and Signal Processing, vol. 43, no. 1-2, pp. 90-102, 2014.

[22] D. J. Rodrigues, A. R. Champneys, M. I. Friswell, and R. E. Wilson, "Two-plane automatic balancing: a symmetry breaking analysis," International Journal of Non-linear Mechanics, vol. 46, no. 9, pp. 1139-1154, 2011.

[23] X. Pan, X. He, K. Wei, H. Wu, J. Gao, and Z. Jiang, "Performance analysis and experimental research of electromagnetic-ring active balancing actuator for hollow rotors of machine tool spindles," Applied Sciences, vol. 9, no. 4, p. 692, 2019.

[24] F. H. Steve, E. A. Russell, and W. Wayne, Aircraft with Transient-Discriminating Propeller Balancing System, US Patents, Alexandria, VA, USA, 2009.

[25] X. Cao, L. F. Chen, and J. J. Gao, "Study of bi-disc electromagnetic balancer movement control for rotor auto-balancing," Journal of Beijing University of Chemical Technology (Natural Science Edition), vol. 37, no. 4, pp. 121-125, 2010.

[26] L. F. Chen, H. Q. Wu, W. M. Wang, and J. J. Gao, "A study of an error-free movement control algorithm for a bi-disc balancer," Journal of Beijing University of Chemical Technology (Natural Science Edition), vol. 39, no. 2, pp. 89-94, 2012. 